\title{
Dysregulation of long non-coding RNA profiles in human colorectal cancer and its association with overall survival
}

\author{
LEI YANG ${ }^{1}$, LINGLING XU ${ }^{2}$, QIAN WANG ${ }^{2}$, MIN WANG $^{2}$ and GUANGYU AN ${ }^{2}$ \\ ${ }^{1}$ Medical Research Center; ${ }^{2}$ Department of Oncology, Beijing Chaoyang Hospital, \\ Capital Medical University, Beijing 100020, P.R. China
}

Received June 4, 2015; Accepted August 25, 2016

DOI: $10.3892 / \mathrm{ol} .2016 .5138$

\begin{abstract}
Long non-coding RNAs (lncRNAs) emerged as key regulators of diverse roles during colorectal cancer (CRC) carcinogenesis, but their specific function still remains to be explored. The present study aimed to re-annotate the Affymetrix Human Exon 1.0 ST Array for defining differential lncRNAs in CRC. Their prognostic relevance was also developed for screening key regulators in CRC. The CRC datasets E-GEOD-31737, E-MATB-829, Affymetrix colon cancer dataset and E-GEOD-24550 were re-purposed for searching differential lncRNAs and exploring their association with overall survival (OS). The identified lncRNAs were validated in CRC tissues or cell lines. As a result, 462,286 and 166 differential lncRNAs were identified, respectively, in three predictive datasets. Among them, 48 lncRNAs were commonly observed to exhibit differential expression in the three datasets. Notably, the overexpression of family with sequence similarity 83 member H (FAM83H)-antisense (AS) $1(\mathrm{P}=0.038)$ and VPS9 domain containing 1 (VPS9D1)-AS1 $(\mathrm{P}=0.020)$ indicated shorter OS time than lower expression. The overexpression of FAM83H-AS1 $(\mathrm{P}=0.033)$ and VPS9D1-AS1 $(\mathrm{P}=0.011)$ was validated in cancerous tissues. Thus, FAM83H-AS1 and VPS9D1-AS1 may potentially enhance carcinogenesis or may be developed as prognostic biomarkers for CRC. In
\end{abstract}

Correspondence to: Dr Lei Yang, Medical Research Center, Beijing Chaoyang Hospital, Capital Medical University, 8 Gongti South Road, Chaoyang, Beijing 100020, P.R. China

E-mail: y16649084@163.com

Professor Guangyu An, Department of Oncology, Beijing Chaoyang Hospital, Capital Medical University, 8 Gongti South Road, Chaoyang, Beijing 100020, P.R. China

E-mail: anguangyu@hotmail.com

Abbreviations: CRC, colorectal cancer; lncRNA, long non-coding RNA; HOTAIR, homeobox transcript antisense intergenic RNA; RT-qPCR, reverse transcription-quantitative polymerase chain reaction; GLM, generalized linear model; OS, overall survival

Key words: colorectal cancer, long non-coding RNA, microarrays, prognosis conclusion, a total of 48 CRC-related lncRNAs were identified, the majority of which were confirmed to exhibit dysregulation. FAM83H-AS1 and VPS9D1-AS1 could have a potential use as prognostic biomarkers for CRC patients.

\section{Introduction}

Colorectal cancer (CRC) is a malignant disease that originates from colorectal epithelial cells (1), and is one of the most commonly diagnosed malignancies in the world (2). Genetic events such as rare or high-penetrance variants in the CRC susceptibility genes and DNA mismatch repair genes have been demonstrated to be important in the etiology of both sporadic and familial CRC (3). However, the carcinogenic mechanisms can only be explained in $<6 \%$ of all CRC cases (3). Therefore, there are still numerous genetic events associated with dysregulation or mutations in CRC patients that remain to be determined.

Long non-coding RNAs (lncRNAs) are a class of transcripts longer than 200 nucleotides without coding potential to be translated into proteins (4). Numerous studies have revealed that lncRNAs were frequently dysregulated in various diseases and had multiple functions in a wide range of pathological processes, including apoptosis, proliferation and invasion-metastasis of malignant tumors $(5,6)$. For instance, colorectal cancer associated transcript 2 was identified by Ling et al as a lncRNA mapping to 8q24 that promoted metastatic progression in CRC (7). Another lncRNA, homeobox transcript antisense intergenic RNA (HOTAIR) has been determined to exhibit higher levels in the plasma of CRC patients than in healthy controls, and its overexpression predicted unfavorable prognosis (8). The association between prognosis of CRC patients and expression of prostate cancer associated transcript 1 and metastasis associated lung adenocarcinoma transcript 1 has also been explored $(9,10)$. The above studies indicated that IncRNAs are important in the regulation of carcinogenesis in CRC, and that lncRNAs could be used as biomarkers of diagnosis and prognosis, and could be potential therapy targets for novel antitumor drugs. However, the function and dysregulation of lncRNAs in CRC still remain to be explored. Thus, the identification of differential lncRNA profiles in CRC is required.

Array-based expression profiles regarding CRC have been established (11). However, these previous array-based profiles 
only compared protein coding RNAs and somatic genomic alteration profiles, such as somatic copy number alteration (11). In addition, those array-based data contained extensive information about IncRNA profiles, which, however, were not explored, since lncRNAs were not the intended targets of study of the original array design. Microarray probes thus can be re-annotated for interrogating lncRNA expression (12), and it is possible to build CRC IncRNA profiles based on those published array-based datasets. The present study aimed to build CRC IncRNA profiles from published Affymetrix Human Exon 1.0 ST arrays (Affymetrix, Inc., Santa Clara, CA, USA). The differential lncRNA expression profiles from three CRC-related datasets were explored, including 44 tumor samples, and the results were validated in another CRC array-based dataset that comprised 166 CRC patients. The expression of those IncRNAs that were significantly associated with prognosis was further determined in CRC cells and cancer tissues.

\section{Materials and methods}

Microarray data. E-GEOD-31737 consisted of 20 paired CRC and adjacent normal tissues; E-MATB-829 contained 14 paired tissues; and E-GEOD-24550 included 166 samples from CRC patients with detailed information about overall survival (OS) time. Data were downloaded from ArrayExpress (http://www.ebi.ac.uk/arrayexpress/). The Affymetrix colon cancer dataset was downloaded from http://www.affymetrix. com/support/technical/sample_data/exon_array_data.affx and comprised 10 paired CRC tissues. All raw CEL files of the above datasets were obtained for exploring underlying lncRNAs. E-GEOD-31737, E-MATB-829 and the Affymetrix datasets were used as experimental sets to identify differentially expressed lncRNAs in CRC, while E-GEOD-24550 was used as a validation set to screen IncRNAs associated with OS rates.

Re-annotation of Affymetrix Exon 1.0 ST Array lncRNA probes. The microarray data were preprocessed with a preprocessing program and re-annotated with Affymetrix CEL file (Affymetrix, Inc.) from noncoder (http://noncoder. mpi-bn.mpg.de/\#) (13). The data were normalized by MAS5.0 (included in the tools of noncoder) prior to lncRNAs annotation. The alignment transcript cluster was filtered by the following steps: i) Genes with $>3$ probes were retained; and ii) probes were mapped to known lncRNAs in NONCODE v3.0 (which was included in the tools of noncoder) (14). Paired $t$-test analysis was used to obtain probe sets whose magnitude of change in expression of lncRNAs between CRC tissue and adjacent normal tissues was significant. Genes with adjusted $\mathrm{P}<0.05$ and fold-change either greater or lower than 2-fold were considered to be differentially expressed. The differentially expressed genes of each dataset were plotted in $\mathrm{R}$ version 3.1.1 (https://www.r-project.org/) using the 'pheatmap' package, which can be accessed via the above link.

Identifying differential lncRNAs associated with OS in CRC. E-GEOD-24550 was used to screen differential lncRNAs associated with the OS rate. The transcript clusters that matched $>3$ probes were retained, and the differential lncRNAs that were determined in the E-GEOD-31737, E-MATB-829 and Affymetrix datasets were further selected.

E-GEOD-24550 provided prognostic information for each patient. Statistical analyses were also performed using $\mathrm{R}$ package version 3.1.1. Time-dependent receiver operating curve (ROC) was applied to calculate the best cutoff values for survival analysis (15). The lncRNAs were further examined with the Kaplan-Meier log-rank test using the $\mathrm{R}$ survival package. The survival curves were plotted with R software.

Tissue samples collection, cell culture and total RNA extraction. Cancerous and paired non-cancerous tissues were consecutively collected from 20 patients with CRC who underwent curative resection of the tumor at Beijing Chaoyang Hospital (Beijing, China) from January 1, 2013 to March 2, 2013. These patients were not subjected to any preoperative chemotherapy or radiotherapy. The freshly dissected tissues were immediately frozen in liquid nitrogen and stored at $-80^{\circ} \mathrm{C}$. RNA was extracted from the CRC samples using the mirVana ${ }^{\mathrm{TM}}$ PARIS ${ }^{\mathrm{TM}}$ kit for protein and RNA isolation (Ambion; Thermo Fisher Scientific, Inc., Waltham, MA, USA) according to the manufacturer's protocol. The study protocol was approved by the Medical Ethics Committee of Chaoyang Hospital of Capital Medical University (Beijing, China), and informed consent was obtained from each subject.

The RKO,HCT116 and HT29 cell lines were purchased from the American Type Culture Collection (Manassas, VA, USA), while the SW620 and SW480 cell lines were obtained from the Culture Collection of the Chinese Academy of Sciences (Shanghai, China). Cells were cultured with complete Dulbecco's modified Eagle medium (Gibco; Thermo Fisher Scientific, Inc.) with $10 \%$ fetal bovine serum (Gibco; Thermo Fisher Scientific, Inc.) and $1 \%$ penicillin-streptomycin (HyClone; GE Healthcare Life Sciences, Logan, UT, USA). Human umbilical vein endothelial cells (HUVECs) were purchased from ScienCell Research Laboratories, Inc. (Carlsbad, CA, USA). Total RNA from the cell lines was extracted with TRIzol (Thermo Fisher Scientific, Inc.). Briefly, cell pellets were resuspended in $500 \mu \mathrm{l}$ TRIzol, and subsequently, chloroform and isopropanol were added for RNA extraction. Finally, total RNA was dissolved with RNase-free water. The concentration of RNA was determined using the NanoDrop 2000 spectrophotometer (NanoDrop Technologies; Thermo Fisher Scientific, Inc., Wilmington, DE, USA).

Reverse transcription (RT)-polymerase chain reaction $(P C R)$ and RT-quantitative (q) PCR. A total of $1 \mu \mathrm{g}$ RNA from each of the samples or cell lines was individually reversed transcribed to synthesize complementary DNA using TranScript II Two-Step RT-PCR SuperMix (Beijing Transgen Biotech Co., Ltd., Beijing, China), and DNase I (Beijing Transgen Biotech Co., Ltd.) was used to degrade the genomic DNA. The primers for VPS9 domain containing 1 (VPS9D1)-antisense (AS) 1 were as follows: Forward primer, 5'-GCTTCAGGCGTGTTTTCCC-3' and reverse primer, 5'-CCCAGAGGCCTTTTCCGTT-3'. The primers for family with sequence similarity 83 member H (FAM83H)-AS1 were as follows: Forward primer, 5'-CCGAGCGAGGAT ATTGAG-3' and reverse primer, 5'-AACACCAACATC AGAGACC-3'. The primers for glyceraldehyde 3-phosphate 


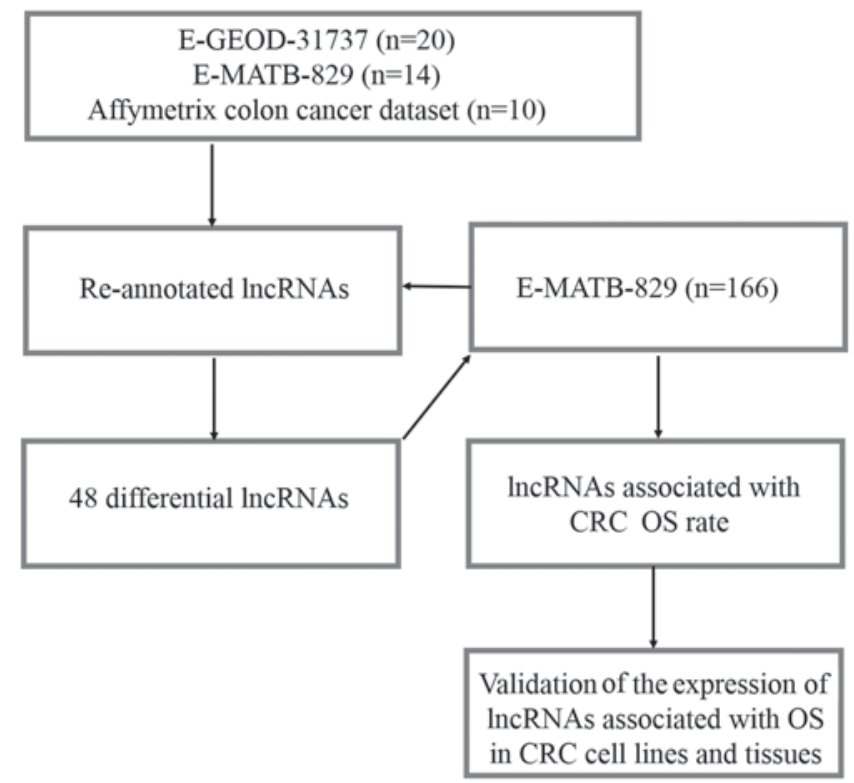

Figure 1. Affymetrix human exon array probe re-annotation and validation pipeline for lncRNAs carried out in the present study. lncRNA, long non-coding RNA; CRC, colorectal cancer; OS, overall surivival.

A

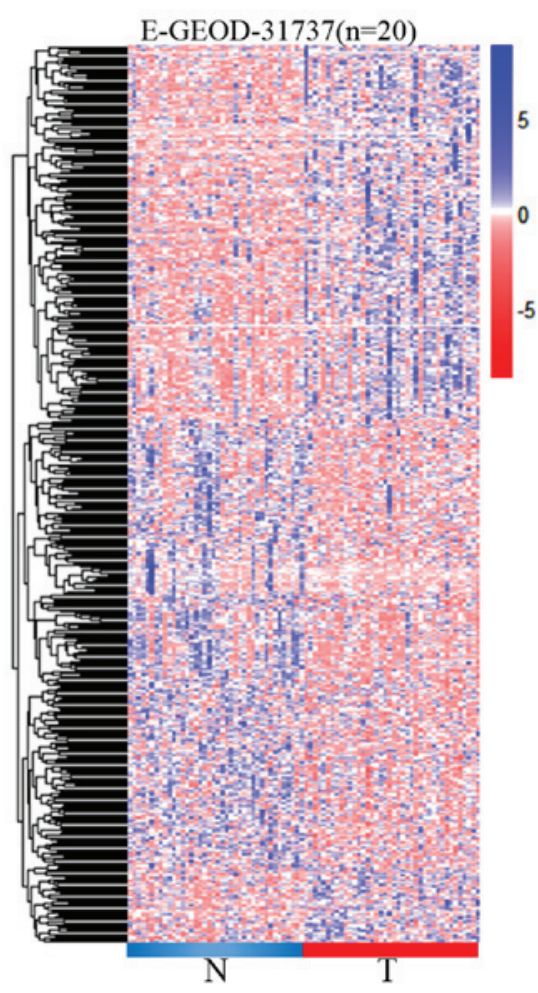

B

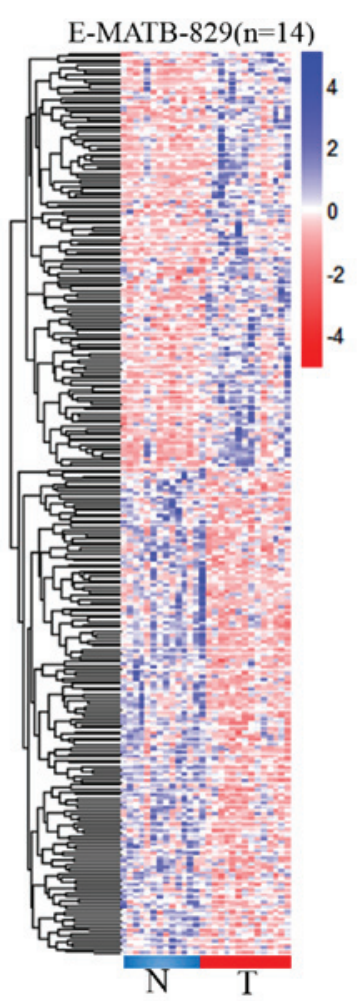

C

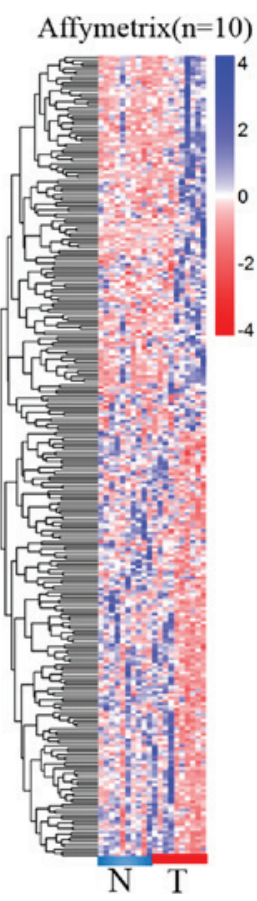

Figure 2. Heat map showing the expression profiles of lncRNAs in three Affymetrix Human Exon 1.0 ST arrays. (A) A total of 462 differential lncRNAs were identified in E-GEOD-31737, which included 20 paired cancerous and non-cancerous CRC samples. (B) A total of 286 differential lncRNAs were identified from 14 paired CRC samples in E-MATB-829. (C) The Affymetrix colon cancer dataset contained 10 paired CRC samples, and 166 differential lncRNAs were selected. N, normal; T, tumor; lncRNA, long non-coding RNA; CRC, colorectal cancer.

dehydrogenase (GAPDH) were as follows: Forward primer, 5'-AATCCCATCACCATCTTCCA-3' and reverse primer, 5'-TGGACTCCACGACGTACTCA-3'. RT-qPCR was performed using a SYBR Premix Ex Taq ${ }^{\mathrm{TM}}$ II kit (Takara Biotechnology Co., Ltd., Dalian, China) with the corresponding sense and antisense primers. The RT-qPCR protocol consisted of an initial denaturation step at $95^{\circ} \mathrm{C}$ for $10 \mathrm{~min}$, followed by 40 cycles of $95^{\circ} \mathrm{C}$ for $10 \mathrm{sec}, 60^{\circ} \mathrm{C}$ for $34 \mathrm{sec}$ and $72^{\circ} \mathrm{C}$ for $10 \mathrm{sec}$. The quantification cycle $(\mathrm{Cq})$ was determined, and the relative IncRNA expression was calculated using the $2^{-\Delta \mathrm{C} q}$ method (16)described by the manufacturer, using GAPDH as the calibrator gene. The diagram of $2^{-\Delta C q} \operatorname{lncRNA}$ relative expression was plot, and the difference between $2^{-\mathrm{ACq}}$ lncRNA relative expression in normal and cancer tissues was 
A

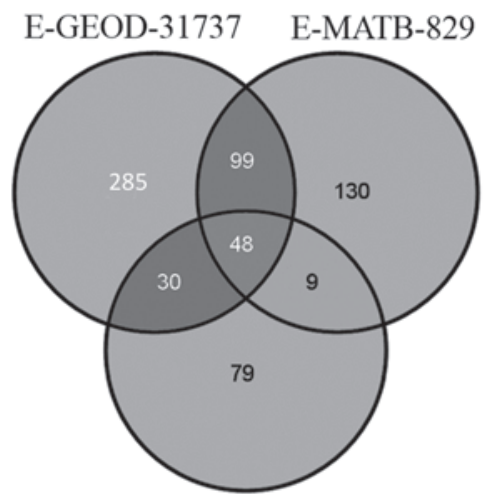

Affymetrix
B

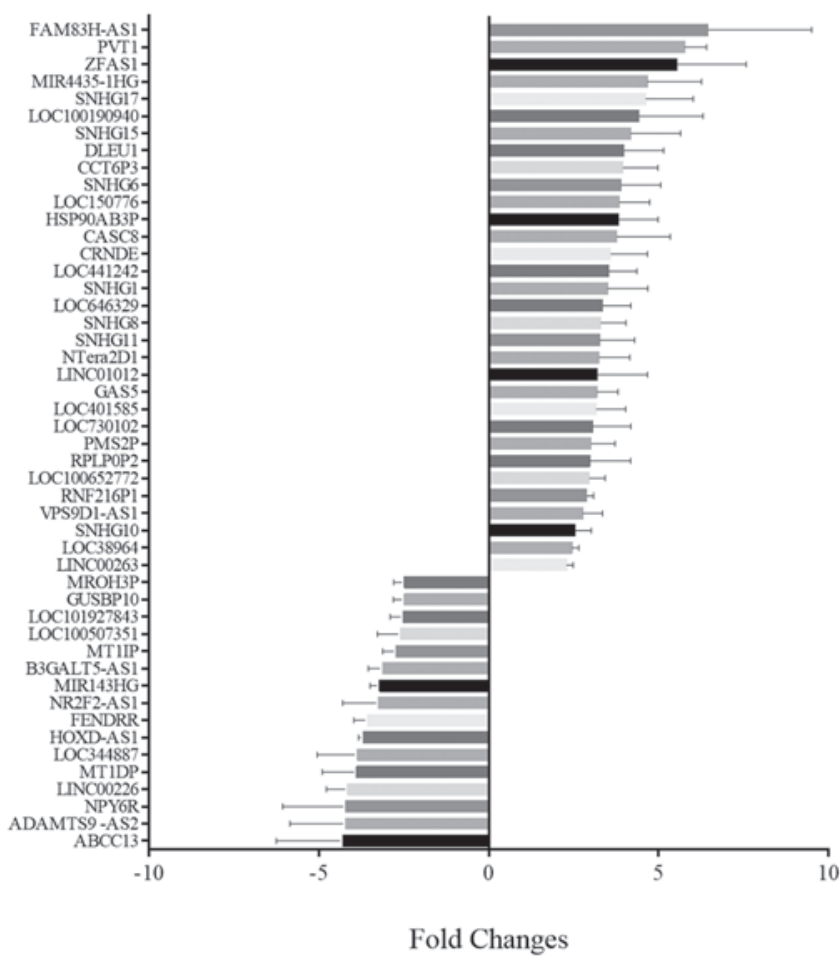

Figure 3. (A) Venn diagram comparing the screened differential lncRNAs from three datasets, of which, 48 lncRNAs were detected in the three datasets. (B) Mean relative fold-changes of these 48 differential lncRNA in the three datasets. lncRNA, long non-coding RNA.

A

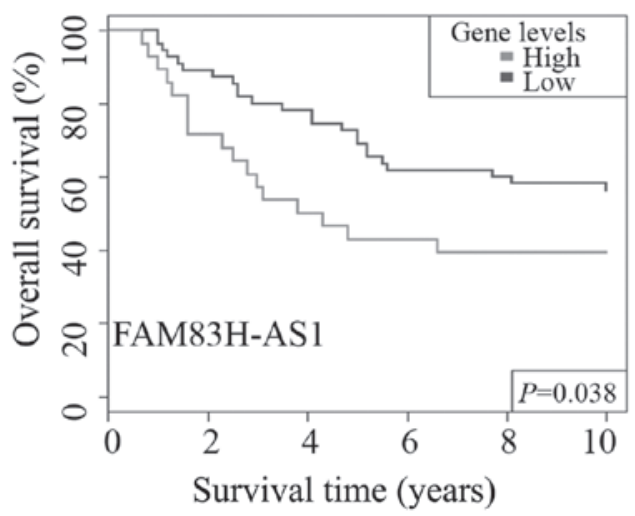

B

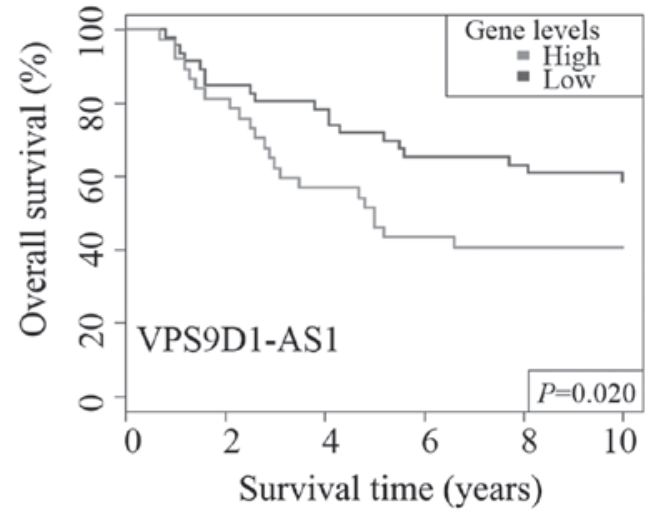

Figure 4. The E-GEOD-24550 dataset was used to validate the association of 48 lncRNAs with the overall survival rate in CRC patients, and two lncRNAs were identified to have the potential to be prognostic biomarkers. The high levels of (A) FAM83H-AS1 and (B) VPS9D1-AS1 in cancer tissues indicated that CRC patients would have poor prognosis. IncRNA, long non-coding RNA; CRC, colorectal cancer; FAM83H, family with sequence similarity 83 member H; AS, antisense; VPS9D1, VPS9 domain containing 1.

calculated with a two-tailed non-parametric Mann-Whitney U test using GraphPad Prism 6.0 software (GraphPad Software, Inc., La Jolla, CA, USA). $\mathrm{P}<0.05$ was considered to indicate a statistically significant difference.

\section{Results}

Differentially expressed lncRNAs in CRC. A pipeline was designed to re-annotate and validate the lncRNAs from four different Affymetrix arrays associated with CRC (Fig. 1). A total of 462 differential lncRNAs were determined from E-GEOD-31737. Among them, 229 lncRNAs were downregulated by $>2$-fold in cancerous tissues $(\mathrm{P}<0.05)$ compared with paired normal tissues. Meanwhile, 233 lncRNAs were noticed to be upregulated in cancerous tissues with $>2$-fold-change in expression and $\mathrm{P}<0.05$ (Fig. 2A). Furthermore, 286 differential lncRNAs were detected in the E-MATB-829 dataset, including 153 downregulated and 133 upregulated lncRNAs, with $>2$-fold-change in expression and $\mathrm{P}<0.05$ (Fig. 2B). Another CRC dataset downloaded from the Affymetrix website was observed to contain 166 differential lncRNAs with $>2$-fold-change in expression and $\mathrm{P}<0.05$, including 78 downregulated and 88 upregulated lncRNAs in cancerous tissues (Fig. 2C). 
A

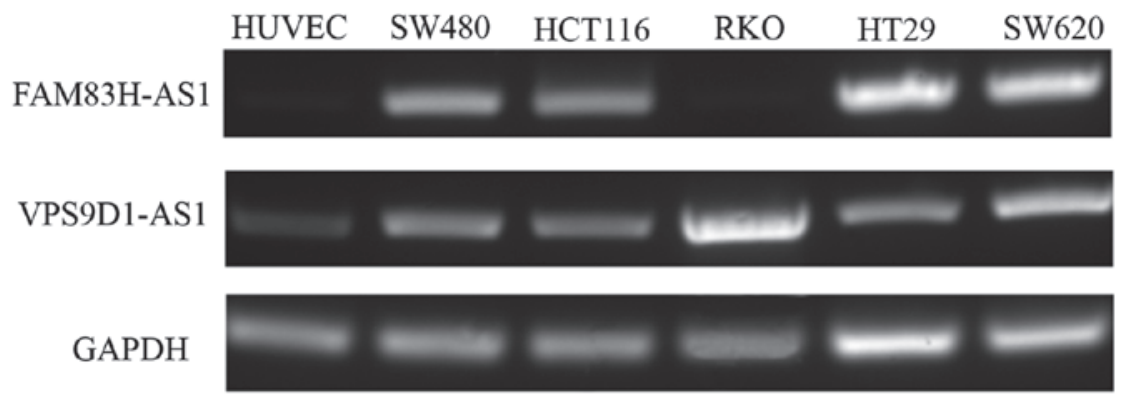

B

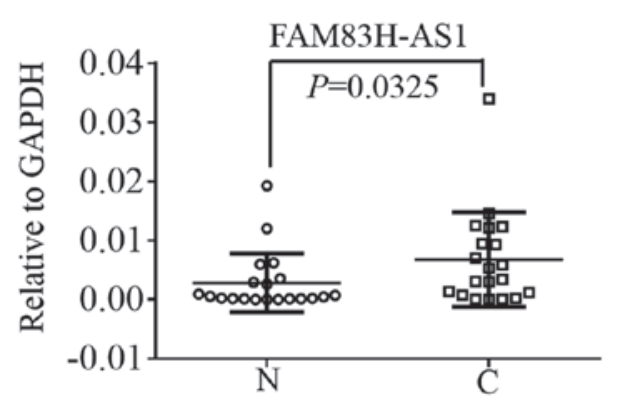

C

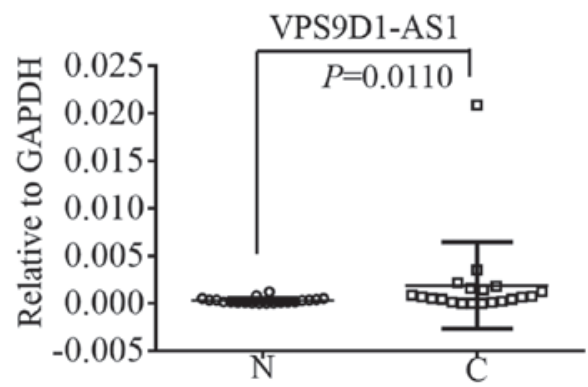

Figure 5. (A) RT-PCR was used to detect the expression of FAM83H-AS1 and VPS9D1-AS1 in HUVEC, SW480, HCT116, RKO, HT29 and SW620 cells. GAPDH was used as the internal reference gene. RT-quantitative PCR was used to validate the expression of (B) FAM83H-AS1 and (C) VPS9D1-AS1 in cancerous and paired non-cancerous tissues from 20 colorectal cancer patients. The paired $t$-test was used to compare the statistical difference of $2^{-\Delta C q}$ between tumor and non-cancerous tissues. GAPDH was used as the internal reference gene. N, normal; C, cancer; GAPDH, glyceraldehyde 3-phosphate dehydrogenase; HUVEC, human umbilical vein endothelial cell; FAM83H, family with sequence similarity 83 member H; AS, antisense; VPS9D1, VPS9 domain containing 1; RT-PCR, reverse transcription-polymerase chain reaction.

Comparison of differential IncRNA expression in three datasets. Venn diagrams were applied for comparative analysis of the differential lncRNAs identified in the above three datasets, as shown in Fig. 3A. A total of 48 differential 1ncRNAs were commonly detected in the three datasets, including 16 downregulated lncRNAs (whose mean values ranged from -4.31 to -2.49 , based on calculating their fold-changes in the three datasets) and 32 upregulated lncRNAs, with mean values ranging from 2.29 to 6.46 (Fig. 3B).

Screening IncRNAs associated with CRC prognosis in E-GEOD-24550. The 48 differential transcript clusters identified in the above three datasets were further validated in E-GEOD-24550. Time-dependent ROC curve applied for setting the best cutoff values, in addition to Kaplan-Meier survival rate, were used to analyze the effects of their overexpression on CRC survival rate. CRC patients were divided into two groups according to best cutoff values of their normalized values. Kaplan-Meier analysis was used to explore the association of lncRNAs with survival rate. Among them, there were two lncRNAs that exhibited significant association with CRC OS rate. FAM83H-AS1 was determined to have a significant association with CRC OS rate $(\mathrm{P}=0.038$, Fig. 4A).The overexpression of VPS9D1-AS1 was associated with CRC shorter OS time ( $\mathrm{P}=0.020$, Fig. $4 \mathrm{~B})$, and indicated a poor prognosis. Thus, both lncRNAs had the potential to be CRC prognostic biomarkers.

Validation of lncRNAs in human CRC cell lines and tissues. The present study next validated the lncRNAs that appeared to be associated with OS in human CRC cell lines and tissues. Firstly, specific primers were designed to clone their sequence from six CRC cell lines, including HUVEC, SW480, HCT116, RKO, HT29 and SW620. Agarose gel electrophoresis analysis revealed that $\mathrm{CRC}$ cell lines had relative higher levels of both FAM83H-AS1 and VPS9D1-AS1 IncRNAs in comparison with HUVECs (Fig. 5A). With the exception of RKO cells, the cell lines HCT116, SW480, HT29 and SW620 expressed FAM83H-AS1, while the expression of FAM83H-AS1 could not be detected in HUVECs. VPS9D1-AS1 was observed to be expressed in all CRC cell lines at higher levels than in HUVECs. In addition, cancerous tissues and paired non-cancerous tissues were collected from 20 patients with CRC to determine the levels of FAM83H-AS1 and VPS9D1-AS1 by RT-qPCR. The relative expression of FAM83H-AS1 in cancerous tissues was demonstrated to be 1.5 -fold higher in 16 (80\%) CRC patients compared with paired non-cancerous tissues. The mean fold-change of FAM83H-AS1 was 62.00. In addition, the results of a paired $t$-test confirmed that the $2^{-\Delta \Delta C q}$ values of FAM83H-AS1 (relative to GAPDH) were significantly higher in CRC tissues $(\mathrm{P}=0.0325)$ than in normal tissues (Fig. 5B). Furthermore, there were 13 (65\%) CRC patients with $>1.5$ fold-change in VPS9D1-AS1 expression, with a mean value of 41.34, ranging from 0.14 to 584.07 in cancerous tissues. The $2^{-\Delta \Delta \mathrm{Cq}}$ values of VPS9D1-AS1 also were observed to be significantly higher in cancerous tissues compared with normal tissues ( $\mathrm{P}=0.0110$, Fig. $5 \mathrm{C})$.

\section{Discussion}

Considering that protein-coding genes only comprise $2 \%$ of the human genome, the majority of transcripts of human or mammalian genomes have lost the potential to be translated into proteins, and a large proportion of them are ncRNAs (17). 
For the past few years, RNA sequencing or array-based strategies have been used to search lncRNAs transcribed from the human genome $(18,19)$. However, those strategies have an expensive cost. Thus, the re-purposing of microarray probes for constructing lncRNA expression profiles in patients with cancer is a cost-effective approach that has been employed by numerous researchers (20). The Affymetrix Exon 1.0 ST Array contains almost 5.4 million probes and is designed to determine the expression of each of the exons of a gene individually (13). In that array, all isoforms of a gene are combined to be a 'transcript cluster', and each exon of the transcript cluster is defined as a 'probe set' (13). In a previous study, each of the four probes designed to match a gene represented the expression of the gene (13). Various other microarray-based platforms could also be re-annotated for lncRNAs, such as Affymetrix U133A $(21,22)$. GATExplorer (http://bioinfow.dep. usal.es/xgate/principal.php) and ncFANs (http://english.ict. cas.cn/) re-annotated several microarrays from Affymetrix for protein and lncRNAs, and revealed that the Affymetrix Exon 1.0 ST Array contains considerably more probes matching lncRNAs than other microarray platforms $(23,24)$. That was the reason why these datasets were collected from previous studies based on the Affymetrix Exon 1.0 ST Array.

In the present study, 462, 286 and 166 differential lncRNAs were identified, respectively, in CRC tissues based on three Exon 1.0 ST databases. A number of the identified lncRNAs had been demonstrated by previous studies to exhibit dysregulated levels in several types of cancer (13). Among them, HoxA transcript at the distal tip RNA antisense RNA (HOTTIP), has been reported to display dysregulated levels in cancerous tissues and to be involved in hepatocarcinogenesis (25). In the present study, HOTTIP was detected to exhibit dysregulation in CRC cancer tissues (E-GEOD-31737). Additionally, the variance transcripts of HOTAIR, including HOTAIRM1, were determined to exhibit differential levels in CRC cancer tissues (E-GEOD-31737 and E-MATB-829) (8). However, variances associated with measurement error were not comparable across different studies (26). In the present study, it was observed that large samples created a large number of differential lncRNAs, and 48 differentially expressed lncRNAs were detected in all three datasets. It may be necessary to further explore more lncRNAs with differential expression in CRC tissues; however those 48 lncRNAs identified in the present study have the most credible probability to be tumor suppressor or oncogenic genes during the process of tumorigenesis.

To more correctly screen CRC-related lncRNAs, 48 differential lncRNAs were validated in other datasets with larger samples. Future studies may be based in those lncRNAs. Furthermore, the present study has identified for the first time two lncRNAs that have the potential to be prognostic biomarkers for CRC patients. These lncRNAs were initially demonstrated to be overexpressed in tumor tissues by three datasets, and the results revealed that they could promote tumor progression. The validation in CRC cell lines or tissues also confirmed that those lncRNAs had higher levels in cancer cell lines and tissues than in normal cell lines or tissues. Further studies should be focused on these lncRNAs for exploring their detailed mechanism of promotion of CRC development.

In conclusion, the current study employed bioinformatics methods and screened 462 differential lncRNAs in CRC. A total of 48 lncRNAs were confirmed by three datasets based on Exon 1.0 ST arrays. Another array-based dataset allowed us to screen two lncRNAs possessing the potential to be prognostic biomarkers of CRC patients. In addition, these two lncRNAs were validated to exhibit higher levels in cancerous tissues in comparison with paired non-cancerous tissues from CRC patients. Those primary findings supported the idea that these two IncRNAs (FAM83H-AS1 and VPS9D1-AS1) may be the key regulators for controlling CRC carcinogenesis and development, and it may be urgent to validate their levels in a large cohort for exploring their clinical significance and identifying their mechanisms of action in CRC.

\section{Acknowledgements}

The present study was supported by Beijing Natural Science Foundation (Beijing, China; grant no. 7154199) and National High Technology Research and Development Program (Beijing, China; grant no. 2012AA02A506).

\section{References}

1. Ogino S, Chan AT, Fuchs CS and Giovannucci E: Molecular pathological epidemiology of colorectal neoplasia: An emerging transdisciplinary and interdisciplinary field. Gut 60: 397-411, 2011.

2. Siegel R, Desantis C and Jemal A: Colorectal cancer statistics, 2014. CA Cancer J Clin 64: 104-117, 2014.

3. Jia WH, Zhang B, Matsuo K, Shin A, Xiang YB, Jee SH, Kim DH, Ren Z, Cai Q, Long J, et al: Genome-wide association analyses in East Asians identify new susceptibility loci for colorectal cancer. Nat Genet 45: 191-196, 2013.

4. Yuan JH, Yang F, Wang F, Ma JZ, Guo YJ, Tao QF, Liu F, Pan W, Wang TT, Zhou CC, et al: A long noncoding RNA activated by TGF- $\beta$ promotes the invasion-metastasis cascade in hepatocellular carcinoma. Cancer Cell 25: 666-681, 2014.

5. Mercer TR, Dinger ME and Mattick JS: Long non-coding RNAs: Insights into functions. Nat Rev Genet 10: 155-159, 2009.

6. Prensner JR, Iyer MK, Sahu A, Asangani IA, Cao Q, Patel L, Vergara IA, Davicioni E, Erho N, Ghadessi M, et al: The long noncoding RNA SChLAP1 promotes aggressive prostate cancer and antagonizes the SWI/SNF complex. Nat Genet 45: 1392-1398, 2013.

7. Ling H, Spizzo R, Atlasi Y, Nicoloso M, Shimizu M, Redis RS, Nishida N, Gafà R, Song J, Guo Z, et al: CCAT2, a novel noncoding RNA mapping to $8 \mathrm{q} 24$, underlies metastatic progression and chromosomal instability in colon cancer. Genome Res 23: 1446-1461, 2013.

8. Svoboda M, Slyskova J, Schneiderova M, Makovicky P, Bielik L, Levy M, Lipska L, Hemmelova B, Kala Z, Protivankova M, et al: HOTAIR long non-coding RNA is a negative prognostic factor not only in primary tumors, but also in the blood of colorectal cancer patients. Carcinogenesis 35: 1510-1515, 2014.

9. Ge X, Chen Y, Liao X, Liu D, Li F, Ruan H and Jia W: Overexpression of long noncoding RNA PCAT-1 is a novel biomarker of poor prognosis in patients with colorectal cancer. Med Oncol 30: 588, 2013.

10. Zheng HT, Shi DB, Wang YW, Li XX, Xu Y, Tripathi P, Gu WL, Cai GX and Cai SJ: High expression of lncRNA MALAT1 suggests a biomarker of poor prognosis in colorectal cancer. Int J Clin Exp Pathol 7: 3174-3181, 2014.

11. Du Z, Fei T, Verhaak RG, Su Z, Zhang Y, Brown M, Chen Y and Liu XS: Integrative genomic analyses reveal clinically relevant long noncoding RNAs in human cancer. Nat Struct Mol Biol 20: 908-913, 2013.

12. Liao Q, Liu C, Yuan X, Kang S, Miao R, Xiao H, Zhao G, Luo H, $\mathrm{Bu} \mathrm{D}$, Zhao H, et al: Large-scale prediction of long non-coding RNA functions in a coding-non-coding gene co-expression network. Nucleic Acids Res 39: 3864-3878, 2011.

13. Gellert P, Ponomareva Y, Braun T and Uchida S: Noncoder: A web interface for exon array-based detection of long non-coding RNAs. Nucleic Acids Res 41: e20, 2013. 
14. Bu D, Yu K, Sun S, Xie C, Skogerbø G, Miao R, Xiao H, Liao Q, Luo H, Zhao G, et al: NONCODE v3.0: Integrative annotation of long noncoding RNAs. Nucleic Acids Res 40 (Database Issue): D210-D215, 2012.

15. Lambert J and Chevret S: Summary measure of discrimination in survival models based on cumulative/dynamic time-dependent ROC curves. Stat Methods Med Res, 2014.

16. Yang M, Tian J, Guo X, Yang Y, Guan R, Qiu M, Li Y, Sun X, Zheng Y, Zhang Y, et al: Long noncoding RNA are aberrantly expressed in human papillary thyroid carcinoma. Oncol Lett 12: 544-552, 2016

17. Batista PJ and Chang HY: Long noncoding RNAs: Cellular address codes in development and disease. Cell 152: 1298-1307, 2013.

18. Ching T, Huang S and Garmire LX: Power analysis and sample size estimation for RNA-Seq differential expression. RNA 20: 1684-1696, 2014.

19. Li J, Chen Z, Tian L, Zhou C, He MY, Gao Y, Wang S, Zhou F, Shi S, Feng X, et al: LncRNA profile study reveals a three-lncRNA signature associated with the survival of patients with oesophageal squamous cell carcinoma. Gut 63: 1700-1710, 2014.

20. Xu X, Zhang Y, Williams J, Antoniou E, McCombie WR, Wu S, Zhu W, Davidson NO, Denoya P and Li E: Parallel comparison of Illumina RNA-Seq and Affymetrix microarray platforms on transcriptomic profiles generated from 5-aza-deoxy-cytidine treated HT-29 colon cancer cells and simulated datasets. BMC Bioinformatics 14 (Suppl 9): S1, 2013
21. Michelhaugh SK, Lipovich L, Blythe J, Jia H, Kapatos G and Bannon MJ: Mining Affymetrix microarray data for long non-coding RNAs: Altered expression in the nucleus accumbens of heroin abusers. J Neurochem 116: 459-466, 2011.

22. Zhang X, Sun S, Pu JK, Tsang AC, Lee D, Man VO, Lui WM, Wong ST and Leung GK: Long non-coding RNA expression profiles predict clinical phenotypes in glioma. Neurobiol Dis 48: $1-8,2012$.

23. Risueno A, Fontanillo C, Dinger ME and De Las Rivas J: GATExplorer: Genomic and transcriptomic explorer; mapping expression probes to gene loci, transcripts, exons and ncRNAs. BMC Bioinformatics 11: 221, 2010.

24. Liao Q, Xiao H, Bu D, Xie C, Miao R, Luo H, Zhao G, Yu K, Zhao H, Skogerb $\varnothing \mathrm{G}$, et al: ncFANs: A web server for functional annotation of long non-coding RNAs. Nucleic Acids Res 39 (Web Server Issue): W118-W124, 2011.

25. Quagliata L, Matter MS, Piscuoglio S, Arabi L, Ruiz C, Procino A, Kovac M, Moretti F, Makowska Z, Boldanova T, et al: Long noncoding RNA HOTTIP/HOXA13 expression is associated with disease progression and predicts outcome in hepatocellular carcinoma patients. Hepatology 59: 911-923, 2014

26. Woo Y, Affourtit J, Daigle S, Viale A, Johnson K, Naggert J and Churchill G: A comparison of cDNA, oligonucleotide, and Affymetrix GeneChip gene expression microarray platforms. J Biomol Tech 15: 276-284, 2004. 